\title{
Electrophilic O-Methylation of a Terminal Nitrosyl Ligand Attained by Early-Late Heterobimetallic Effect
}

\author{
Kazuya Arashiba, ${ }^{\dagger}$ Shoji Matsukawa, ${ }^{\ddagger}$ Shigeki Kuwata, ${ }^{\S}$ Yoshiaki Tanabe, ${ }^{\dagger}$ Masakazu Iwasaki ${ }^{\natural}$ and \\ Youichi Ishii, ${ }^{, \dagger, \#}$ \\ $\dagger$ Department of Applied Chemistry, Faculty of Science and Engineering, \\ Chuo University, Kasuga, Bunkyo-ku, Tokyo 112-8551, Japan \\ $\ddagger$ Institute of Industrial Science,
}

The University of Tokyo, Komaba, Meguro-ku, Tokyo 153-8505, Japan

$\S$ Department of Applied Chemistry, Graduate School of Science and Engineering,

Tokyo Institute of Technology, O-okayama, Meguro-ku, Tokyo 152-8552, Japan

I Department of Applied Chemistry, Faculty of Engineering, Saitama Institute of Technology, Okabe, Saitama 369-0293, Japan

${ }^{*}$ Coordination Chemistry Laboratories,

Institute for Molecular Science, Myodaiji, Okazaki 444-8787, Japan

Table of contents

Experimental Section $\quad$ S2

Table S1. Cyclic voltammetry data for the oxidation of 5-8. S3

Table S2. X-ray Crystallographic Data for $\mathbf{7}, \mathbf{9}^{+} \mathrm{PF}_{6}{ }^{-}$and $\mathbf{1 0}^{+} \mathrm{BPh}_{4}{ }^{-}$. S5

References $\quad$ S6

Figure S1. Molecular structure of 7.

Table S3. Selected bond lengths ( $\AA$ ) and angles (deg) for 7. $\quad$ S7

Figure S2. Molecular structure of the cationic part of $\mathbf{9}^{+} \mathrm{PF}_{6}{ }^{-}$. S8

Table S4. Selected bond lengths $(\AA)$ and angles (deg) for $\mathbf{9}^{+} \mathrm{PF}_{6}{ }^{-}$. $\quad$ S8

Figure S3. Molecular structure of the cationic part of $\mathbf{1 0}^{+} \mathrm{BPh}_{4}{ }^{-}$. S9

Table S5. Selected bond lengths $(\AA)$ and angles (deg) for $\mathbf{1 0}^{+} \mathrm{BPh}_{4}{ }^{-}$. S9 


\section{Experimental Section}

General Remarks. All manipulations were carried out under an atmosphere of nitrogen by using standard Schlenk techniques. $\mathrm{CH}_{2} \mathrm{Cl}_{2}$ and $\mathrm{NEt}_{3}$ were dried and distilled over $\mathrm{P}_{4} \mathrm{O}_{10}$ and $\mathrm{KOH}$, respectively, while the other solvents (dehydrated-grade) were purchased from Aldrich and used as received. $\quad\left[\mathrm{Cp} * \mathrm{M}(\mathrm{SH})_{2}\left(\mathrm{PMe}_{3}\right)\right](\mathbf{1}, \mathrm{M}=\mathrm{Rh} ; \mathbf{2}, \mathrm{M}=\mathrm{Ir})^{1}$ and $\left[\mathrm{Cp}^{*} \mathrm{M}^{\prime} \mathrm{Cl}_{2}(\mathrm{NO})\right]\left(3, \mathrm{M}^{\prime}=\mathrm{Mo} ; \mathbf{4}, \mathrm{M}=\mathrm{W}\right)^{2}$ were prepared according to the literature methods. ${ }^{1} \mathrm{H}(400 \mathrm{MHz})$ and ${ }^{31} \mathrm{P}\left\{{ }^{1} \mathrm{H}\right\}(121 \mathrm{MHz}) \mathrm{NMR}$ spectra were recorded on a JEOL JNM-GSX-400 and a Varian Mercury-300 spectrometers, respectively, by using $\mathrm{CDCl}_{3}$ as the solvent unless otherwise noted. IR spectra were recorded on a JASCO FT/IR-410 spectrometer. Elemental Analyses were performed on a Perkin-Elmer 2400II CHN analyzer.

Preparation of [Cp*M(PMe $\left.\left.{ }_{3}\right)(\mu-S)_{2} M^{\prime}(N O) C^{*}\right]$ (5, $M=R h, M^{\prime}=$ Mo; 6, M = Ir, M' = Mo; 7, $\mathbf{M}=\mathbf{R h}, \mathbf{M}^{\prime}=\mathbf{W} ; \mathbf{8}, \mathbf{M}=\mathbf{I r}, \mathbf{M}^{\prime}=\mathbf{W}$ ). The following procedure for the preparation of [Cp*Rh( $\left.\left.\mathrm{PMe}_{3}\right)(\mu-\mathrm{S})_{2} \mathrm{~W}(\mathrm{NO}) \mathrm{Cp} *\right]$ (7) is representative. To a solution of $\left[\mathrm{Cp}^{*} \mathrm{WCl}_{2}(\mathrm{NO})\right]$ (4: $475 \mathrm{mg}$, $1.13 \mathrm{mmol})$ in THF $(40 \mathrm{~mL})$ at $-40{ }^{\circ} \mathrm{C}$ were added $\left[\mathrm{Cp} * \mathrm{Rh}(\mathrm{SH})_{2}\left(\mathrm{PMe}_{3}\right)\right](\mathbf{1}: 430 \mathrm{mg}, 1.13 \mathrm{mmol})$ and $\mathrm{NEt}_{3}(0.33 \mathrm{~mL}, 2.37 \mathrm{mmol})$, and the mixture was warmed gradually to room temperature. After $3 \mathrm{~h}$ of stirring at room temperature, the dark brown solution was dried up in vacuo, and the residue was dissolved in $\mathrm{CH}_{2} \mathrm{Cl}_{2}$ (15 mL) to load onto an alumina column, where the adsorbed mixture was eluted with THF-hexane (2:1). The green band was collected, and the solvent was removed in vacuo. Recrystallization of the residual dark green solid from benzene-hexane (20 mL-45 mL) afforded 7 as dark green crystals (472 mg, 0.649 mmol, 57\% yield). ${ }^{31} \mathrm{P}\left\{{ }^{1} \mathrm{H}\right\} \mathrm{NMR}: \delta 7.6\left(\mathrm{~d}, \mathrm{PMe}_{3},{ }^{1} J_{\mathrm{RhP}}=147 \mathrm{~Hz}\right)$. ${ }^{1} \mathrm{H}$ NMR: $\delta 1.99$ (s, 15H, Cp*W), 1.92 (d, 15H, Cp*Rh, ${ }^{4} J_{\mathrm{PH}}=2.4 \mathrm{~Hz}$ ), 1.25 (d, 9H, $\mathrm{PMe}_{3},{ }^{2} J_{\mathrm{PH}}=11.0$ $\mathrm{Hz}$ ). IR ( $\mathrm{cm}^{-1}, \mathrm{KBr}$ disk): $1490\left(v_{\mathrm{NO}}\right)$. Anal. Calcd for $\mathrm{C}_{23} \mathrm{H}_{39} \mathrm{NOPRhS}_{2} \mathrm{~W}$ : C, 37.98; H, 5.40; N, 1.93. Found: C, 38.10; H, 5.45; N, 2.00.

5: dark green crystals, 79\% yield. ${ }^{31} \mathrm{P}\left\{{ }^{1} \mathrm{H}\right\} \mathrm{NMR}: \delta 11.2\left(\mathrm{~d}, \mathrm{PMe}_{3},{ }^{1} J_{\mathrm{RhP}}=147 \mathrm{~Hz}\right) .{ }^{1} \mathrm{H}$ NMR: $\delta$ 1.91 (s, 15H, Cp*Mo), 1.89 (d, 15H, Cp*Rh, ${ }^{4} J_{\mathrm{PH}}=2.7 \mathrm{~Hz}$ ), 1.26 (d, 9H, $\mathrm{PMe}_{3},{ }^{2} J_{\mathrm{PH}}=11.0 \mathrm{~Hz}$ ). IR ( $\mathrm{cm}^{-1}, \mathrm{KBr}$ disk): $1515\left(v_{\mathrm{NO}}\right)$. Anal. Calcd for $\mathrm{C}_{23} \mathrm{H}_{39} \mathrm{MoNOPRhS}_{2}$ : C, 43.20; H, 6.15; N, 2.19. Found: C, 43.52; H, 6.19; N, 2.27.

6: dark green crystals, 66\% yield. ${ }^{31} \mathrm{P}\left\{{ }^{1} \mathrm{H}\right\}$ NMR: $\delta-22.8$ (s, $\mathrm{PMe}_{3}$ ). ${ }^{1} \mathrm{H}$ NMR: $\delta 1.97$ (s, 15H, Cp*Mo), 1.89 (d, 15H, Cp*Ir, ${ }^{4} J_{\mathrm{PH}}=1.5 \mathrm{~Hz}$ ), 1.34 (d, 9H, PMe $3{ }^{2} J_{\mathrm{PH}}=11.0 \mathrm{~Hz}$ ). $\quad$ IR ( $\mathrm{cm}^{-1}, \mathrm{KBr}$ disk): $1521\left(v_{\mathrm{NO}}\right)$. Anal. Calcd for $\mathrm{C}_{23} \mathrm{H}_{39} \mathrm{IrMoNOPS}_{2}$ : C, 37.90; H, 5.39; N, 1.92. Found: C, 38.08; H, 5.44; N, 1.97.

8: dark violet crystals, 48\% yield. ${ }^{31} \mathrm{P}\left\{{ }^{1} \mathrm{H}\right\}$ NMR: $\delta-28.6$ (s, $\mathrm{PMe}_{3}$ ). ${ }^{1} \mathrm{H}$ NMR: $\delta 2.02$ (s, 15H, Cp*W), 1.93 (d, 15H, Cp*Ir, $\left.{ }^{4} J_{\mathrm{PH}}=1.7 \mathrm{~Hz}\right), 1.34$ (d, 9H, PMe $3,{ }^{2} J_{\mathrm{PH}}=11.0 \mathrm{~Hz}$ ). $\quad$ IR (cm ${ }^{-1}, \mathrm{KBr}$ disk): $1491\left(v_{\mathrm{NO}}\right)$. Anal. Calcd for $\mathrm{C}_{23} \mathrm{H}_{39} \mathrm{IrNOPS}_{2} \mathrm{~W}$ : C, 33.82; H, 4.81; N, 1.71. Found: C, 33.68; H, 4.86; N, 1.79 .

Preparation of $\left[\mathrm{Cp}^{*} \mathbf{R h}\left(\mathrm{PMe}_{3}\right)(\boldsymbol{\mu}-\mathbf{S})_{2} \mathbf{W}(\mathbf{N O M e}) \mathbf{C p}^{*}\right](\mathbf{O T f})\left(\mathbf{9}^{+} \mathbf{O T f}^{-}\right)$. To a dark green solution of 7 (160 mg, $0.220 \mathrm{mmol})$ in $\mathrm{CH}_{2} \mathrm{Cl}_{2}(10 \mathrm{~mL})$ was added MeOTf $(25 \mu \mathrm{L}, 0.22 \mathrm{mmol})$ at $-40{ }^{\circ} \mathrm{C}$, and the resultant red solution was gradually warmed to room temperature. The solution was stirred 
overnight and was dried in vacuo. The dark red solids were recrystallized from $\mathrm{CH}_{2} \mathrm{Cl}_{2}$-hexane (5 $\mathrm{mL}-10 \mathrm{~mL}$ ) to afford $\mathbf{9}^{+} \mathrm{OTf}^{-}$as a red solid (179 mg, $0.201 \mathrm{mmol}, 91 \%$ yield). ${ }^{31} \mathrm{P}\left\{{ }^{1} \mathrm{H}\right\}$ NMR: $\delta 8.1$ (s, $\mathrm{PMe}_{3},{ }^{1} J_{\mathrm{RhP}}=142 \mathrm{~Hz}$ ). ${ }^{1} \mathrm{H}$ NMR: $\delta 4.06$ (s, 3H, NOMe), 2.18 (s, 15H, Cp*W), 1.93 (d, 15H, Cp*Rh, $\left.{ }^{4} J_{\mathrm{PH}}=2.9 \mathrm{~Hz}\right), 1.31\left(\mathrm{~d}, 9 \mathrm{H}, \mathrm{PMe}_{3},{ }^{2} J_{\mathrm{PH}}=11.2 \mathrm{~Hz}\right) . \mathrm{IR}\left(\mathrm{cm}^{-1}, \mathrm{KBr}\right.$ disk): $1272\left(v_{\mathrm{NO}}\right)$. Anal. Calcd for $\mathrm{C}_{25} \mathrm{H}_{42} \mathrm{~F}_{3} \mathrm{NO}_{4} \mathrm{PRhS}_{3} \mathrm{~W}$ : C, 33.68; H, 4.75; N, 1.57. Found: C, 33.50; H, 4.81; N, 1.54. Single crystals suitable for $\mathrm{X}$-ray crystallography were obtained as $\mathbf{9}^{+} \mathrm{PF}_{6}^{-}$by the anion metathesis of $\mathbf{9}^{+} \mathrm{OTf}^{-}$with ${ }^{\mathrm{n}} \mathrm{Bu}_{4} \mathrm{NPF}_{6}$ and further recrystallization from $\mathrm{CH}_{2} \mathrm{Cl}_{2}$-hexane.

Preparation of $\left[\mathrm{Cp}^{*} \mathrm{Rh}\left(\mathrm{PMe}_{3}\right)(\mu-\mathrm{SMe})(\mu-\mathrm{S}) \mathrm{Mo}(\mathrm{NO}) \mathrm{Cp}^{*}\right]\left(\mathrm{BPh}_{4}\right) \quad\left(\mathbf{1 0}^{+} \mathrm{BPh}_{4}{ }^{-}\right)$. To a dark green solution of 5 (37 mg, $0.058 \mathrm{mmol})$ in $\mathrm{CH}_{2} \mathrm{Cl}_{2}(5 \mathrm{~mL})$ was added MeOTf (6.6 $\left.\mu \mathrm{L}, 0.058 \mathrm{mmol}\right)$ at $-40{ }^{\circ} \mathrm{C}$, and the mixture was stirred at room temperature for $5 \mathrm{~h}$. After the resultant dark red solution was evaporated to dryness in vacuo, $\mathrm{NaBPh}_{4}$ (ca. $20 \mathrm{mg}$ ) and $\mathrm{CH}_{2} \mathrm{Cl}_{2}(5 \mathrm{~mL}$ ) were added to the residual solid. The mixture was filtered and $\mathrm{Et}_{2} \mathrm{O}(10 \mathrm{~mL})$ was added to the concentrated filtrate $(4 \mathrm{~mL})$. Dark red crystals of $\mathbf{1 0}^{+} \mathrm{BPh}_{4}^{-}$were gradually formed on standing at room temperature for two weeks, which were collected by filtration and washed with hexane. (30.0 mg, $0.031 \mathrm{mmol}$, 53\% yield). ${ }^{31} \mathrm{P}\left\{{ }^{1} \mathrm{H}\right\}$ NMR: $\delta 12.5$ (d, $\mathrm{PMe}_{3},{ }^{2} J_{\mathrm{RhP}}=135 \mathrm{~Hz}$ ). ${ }^{1} \mathrm{H}$ NMR: $\delta 2.58$ (s, 3H, SMe), 1.90 (s, 15H, Cp*Mo), 1.74 (d, 15H, Cp*Rh, ${ }^{4} J_{\mathrm{PH}}=3.2 \mathrm{~Hz}$ ), 0.94 (d, 9H, PMe $3,{ }^{2} J_{\mathrm{PH}}=11.5 \mathrm{~Hz}$ ). $\quad$ IR (cm ${ }^{-1}, \mathrm{KBr}$ disk): 1573 $\left(v_{\mathrm{NO}}\right)$. Anal. Calcd for $\mathrm{C}_{48} \mathrm{H}_{62} \mathrm{BMoNOPRhS}_{2}$ : C, 59.20; H, 6.42; N, 1.44. Found: C, 59.07; H, 6.48; N, 1.39 .

Electrochemical Properties of Complexes 5-8. Cyclic voltammetry studies were performed with a BAS CV-50W analyzer. Potentials were measured at a glassy carbon working electrode in 0.1 $\mathrm{M}\left({ }^{\mathrm{n}} \mathrm{Bu}_{4} \mathrm{~N}\right)\left(\mathrm{BF}_{4}\right)$ solution of $\mathrm{CH}_{2} \mathrm{Cl}_{2}$. The oxidation potentials vs SCE $\left(\mathrm{E}_{1 / 2}\right)$ and the peak current ratio $\left(i_{\mathrm{p}, \mathrm{c}} i_{\mathrm{p}, \mathrm{a}}\right)$ are shown in Table S1.

Table S1. Cyclic voltammetry data for the oxidation of dinuclear complexes [Cp*M(PMe $\left.)(\mu-\mathrm{S})_{2} \mathrm{M}^{\prime}(\mathrm{NO}) \mathrm{Cp}^{*}\right]$ (5-8).

\begin{tabular}{ccc}
\hline compound & $E_{1 / 2}{ }^{\mathrm{a}}$ & $i_{\mathrm{p}, \mathrm{c}} i_{\mathrm{p}, \mathrm{a}}$ \\
\hline $\mathbf{5}\left(\mathrm{M}=\mathrm{Rh}, \mathrm{M}^{\prime}=\mathrm{Mo}\right)$ & +0.24 & 1.27 \\
$\mathbf{6}\left(\mathrm{M}=\mathrm{Ir}, \mathrm{M}^{\prime}=\mathrm{Mo}\right)$ & +0.22 & 1.18 \\
$\mathbf{7}\left(\mathrm{M}=\mathrm{Rh}, \mathrm{M}^{\prime}=\mathrm{W}\right)$ & +0.13 & 1.30 \\
$\mathbf{8}\left(\mathrm{M}=\mathrm{Ir}, \mathrm{M}^{\prime}=\mathrm{W}\right)$ & +0.12 & 0.94 \\
\hline a vs SCE, reversible. & &
\end{tabular}

X-ray Diffraction Studies. Diffraction data were collected on a Rigaku AFC-7S four-circle automated diffractometer with graphite-monochromated Mo $K \alpha$ radiation $(\lambda=0.71069 \AA)$ at room temperature using the $\omega-2 \theta$ scan technique at a rate of $16^{\circ} \min ^{-1}$ to a maximum $2 \theta$ value of $55^{\circ}$. Cell parameters were determined by least-squares refinement of 25 machine-centered reflections. Intensity data were corrected for Lorenz-polarization effects and for empirical absorption ( $\psi$ scans). A correction for secondary extinction was further applied for 7 (coefficient, 32.544), and $\mathbf{9}^{+} \mathrm{PF}_{6}{ }^{-}$ 
(coefficient, 52.218). ${ }^{3}$ The intensities of three standard reflections were monitored every 150 reflections during the data collection, showing no significant decay. Structure solution and refinements were carried out by using the CrystalStructure crystallographic software package. ${ }^{4}$ The positions of the heavy atoms were determined by Patterson methods (PATTY) ${ }^{5}$ and the remaining non-hydrogen atoms were found by the subsequent Fourier syntheses. ${ }^{6} \quad$ All non-hydrogen atoms were refined on $F_{0}(I>3 \sigma$ (I)) anisotropically by full-matrix least-square techniques, while all the hydrogen atoms were placed at the calculated positions with fixed isotropic parameter. Details of the X-ray diffraction study are summarized in Table S2. Selected bond lengths and angles are summarized in Tables S3-S5, and their ORTEP drawings are shown in Figures S1-S3. 
Table S2. X-ray Crystallographic Data for $\left[\mathrm{Cp} * \mathrm{Rh}\left(\mathrm{PMe}_{3}\right)(\mu-\mathrm{S})_{2} \mathrm{~W}(\mathrm{NO}) \mathrm{Cp} *\right](7),\left[\mathrm{Cp} * \mathrm{Rh}\left(\mathrm{PMe}_{3}\right)(\mu-\mathrm{S})_{2} \mathrm{~W}-\right.$ (NOMe)Cp*][ $\left.\mathrm{PF}_{6}\right]\left(\mathbf{9}^{+} \mathrm{PF}_{6}{ }^{-}\right),\left[\mathrm{Cp} * \mathrm{Rh}\left(\mathrm{PMe}_{3}\right)(\mu-\mathrm{SMe})(\mu-\mathrm{S}) \mathrm{Mo}(\mathrm{NO}) \mathrm{Cp}^{*}\right]\left[\mathrm{BPh}_{4}\right]\left(\mathbf{1 0}^{+} \mathrm{BPh}_{4}{ }^{-}\right)$.

\begin{tabular}{|c|c|c|c|}
\hline & 7 & $\mathbf{9}^{+} \mathrm{PF}_{6}^{-}$ & $\mathbf{1 0}^{+} \mathrm{BPh}_{4}^{-}$ \\
\hline chemical formula & $\mathrm{C}_{23} \mathrm{H}_{39} \mathrm{NOPRhS}_{2} \mathrm{~W}$ & $\mathrm{C}_{24} \mathrm{H}_{42} \mathrm{~F}_{6} \mathrm{NOP}_{2} \mathrm{RhS}_{2} \mathrm{~W}$ & $\mathrm{C}_{48} \mathrm{H}_{62} \mathrm{BMoNOPRhS}_{2}$ \\
\hline formula weight & 727.42 & 887.42 & 973.77 \\
\hline dimensions of crystals & $0.60 \times 0.30 \times 0.30$ & $0.90 \times 0.30 \times 0.30$ & $0.40 \times 0.40 \times 0.20$ \\
\hline crystal system & monoclinic & monoclinic & triclinic \\
\hline space group & $P 2_{1} / n$ & $P 2_{1} / c$ & $P \overline{1}$ \\
\hline$a, \AA$ & $17.008(2)$ & $8.714(3)$ & $10.204(1)$ \\
\hline$b, \AA$ & $18.843(2)$ & $24.849(4)$ & 18.072(3) \\
\hline$c, \AA$ & $17.127(2)$ & $14.533(2)$ & $27.000(5)$ \\
\hline$\alpha, \operatorname{deg}$ & & & $75.05(1)$ \\
\hline$\beta$, deg & $97.121(8)$ & $91.52(2)$ & $88.46(1)$ \\
\hline$\gamma$, deg & & & 82.92(1) \\
\hline$V, \AA^{3}$ & $5446(1)$ & $3145(1)$ & 4773(1) \\
\hline$Z$ & 8 & 4 & 4 \\
\hline$\rho_{\text {calcd }}, \mathrm{g} \mathrm{cm}^{-3}$ & 1.774 & 1.874 & 1.355 \\
\hline$F(000)$ & 2864.00 & 1744.00 & 2016.00 \\
\hline$\mu, \mathrm{cm}^{-1}$ & 50.57 & 44.71 & 7.62 \\
\hline trans. factors range & $0.7495-1.0000$ & $0.4299-1.0000$ & $0.8174-1.0000$ \\
\hline $2 \theta$ range, deg & $5<2 \theta<55$ & $5<2 \theta<55$ & $5<2 \theta<55$ \\
\hline no. reflections measured & 13308 & 7875 & 22495 \\
\hline no. unique reflections & 12486 & 7213 & 21897 \\
\hline no. reflections used $(I>3 \sigma(I))$ & 8566 & 6041 & 8584 \\
\hline no. parameters refined & 620 & 422 & 1133 \\
\hline$R(I>3 \sigma(I))^{\mathrm{a}}$ & 0.037 & 0.030 & 0.053 \\
\hline$R_{\mathrm{w}}(I>3 \sigma(I))^{\mathrm{b}}$ & 0.038 & 0.031 & 0.054 \\
\hline $\operatorname{GOF}(I>3 \sigma(I))^{\mathrm{c}}$ & 1.012 & 1.018 & 1.014 \\
\hline max diff peak / hole, e $\AA^{-3}$ & $0.81 /-1.60$ & $1.12 /-1.26$ & $0.60 /-0.62$ \\
\hline
\end{tabular}




\section{References}

(1) (a) Klein, D. P.; Kloster, G. M.; Bergman, R. G. J. Am. Chem. Soc. 1990, 112, 2022. (b) Dobbs, D. A.; Bergman, R. G. Inorg. Chem. 1994, 33, 5329.

(2) Dryden, N. H.; Legzdins, P.; Batchelor, R. J.; Einstein, W. B. Organometallics 1991, 10, 2077.

(3) Larson, A. C. In Crystallographic Computing; Proceedings of an International Summer School organized by The Commission on Crystallographic Computing of the International Union of Crystallography and held in Ottawa, 4-11 August 1969; Ahmed, F. R., Hall, S. R., Huber, C. P. Eds.; Munksgaard: Copenhagen, Denmark, 1970; pp. 291-295.

(4) (a) CrystalStructure 3.60: Single Crystal Structure Analysis Software; Rigaku Corp: Tokyo, Japan and MSC: The Woodlands, TX, 2000-2004. (b) Watkin, D. J.; Prout, C. K.; Carruthers, J. R.; Betteridge, P. W. CRYSTALS Issue 10; Chemical Crystallography Laboratory: Oxford, UK, 1996.

(5) Beurskens, P. T.; Admiraal, G.; Beurskens, G.; Bosman, W. P.; Garcia-Granda, S.; Gould, R. O.; Smits, J. M. M.; Smykalla, C. PATTY: The DIRDIF program system; Technical Report of the Crystallography Laboratory, University of Nijmengen: Nijmengen, The Netherlands, 1992.

(6) Beurskens, P. T.; Admiraal, G.; Beurskens, G.; Bosman, W. P.; de Gelder. R.; Israel, R.; Smits, J.; M. M. DIRDIF99: The DIRDIF-99 program system; Technical Report of the Crystallography Laboratory, University of Nijmengen: Nijmengen, The Netherlands, 1999. 


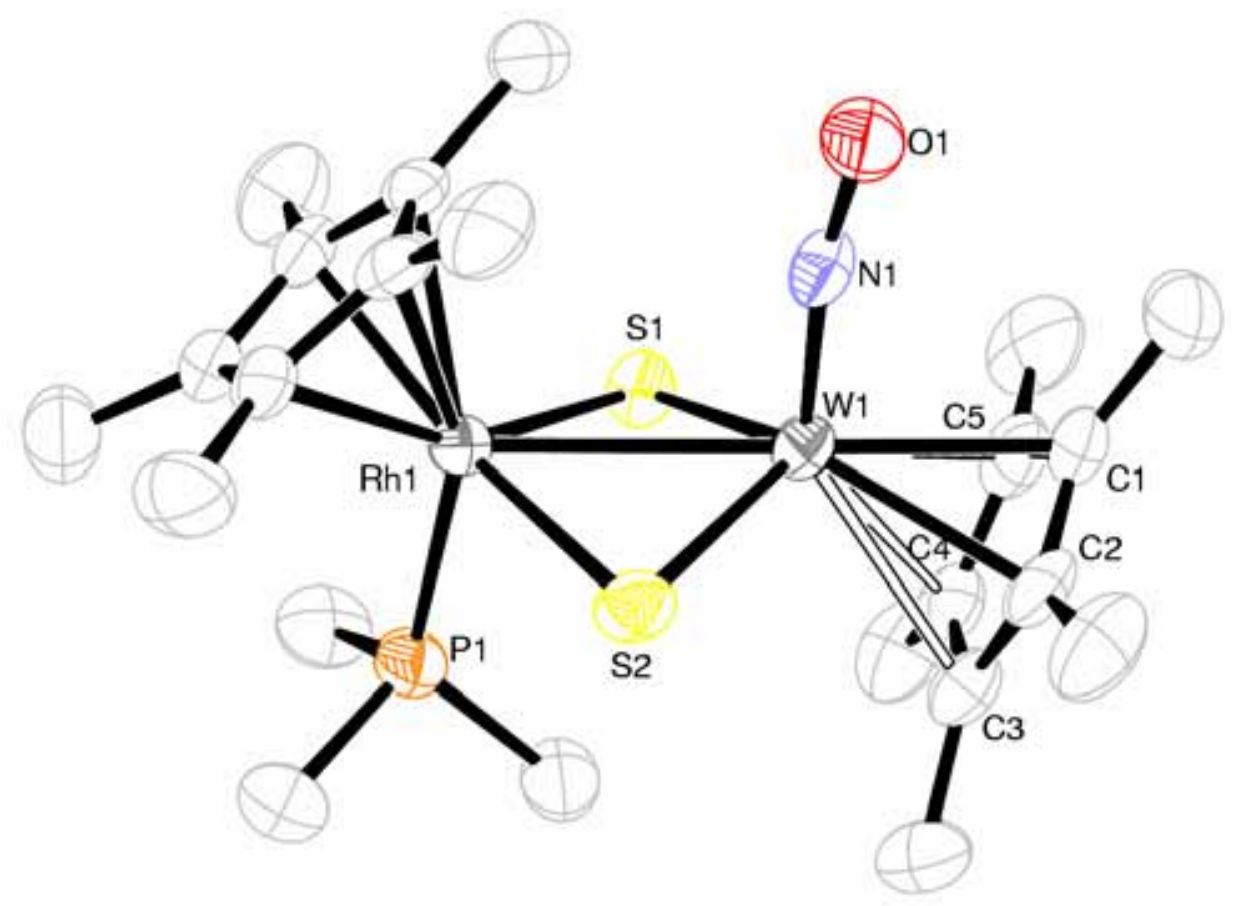

Figure S1. Molecular structure of $\left[\mathrm{Cp} * \mathrm{Rh}\left(\mathrm{PMe}_{3}\right)(\mu-\mathrm{S})_{2} \mathrm{~W}(\mathrm{NO}) \mathrm{Cp} *\right]$ (7). One of the two crystallographically independent molecules. Thermal ellipsoids are shown at the 50\% probability level. Hydrogen atom are omitted for clarity.

Table S3. Selected bond lengths $(\AA)$ and angles (deg) for 7.

\begin{tabular}{llll}
\hline \multicolumn{3}{c}{ molecule 1} & \multicolumn{2}{c}{ molecule 2} \\
\hline $\mathrm{W}(1)-\mathrm{Rh}(1)$ & $2.9149(6)$ & $\mathrm{W}(2)-\mathrm{Rh}(2)$ & $2.8986(6)$ \\
$\mathrm{W}(1)-\mathrm{S}(1)$ & $2.331(2)$ & $\mathrm{W}(2)-\mathrm{S}(3)$ & $2.342(2)$ \\
$\mathrm{W}(1)-\mathrm{S}(2)$ & $2.327(2)$ & $\mathrm{W}(2)-\mathrm{S}(4)$ & $2.330(2)$ \\
$\mathrm{W}(1)-\mathrm{N}(1)$ & $1.754(6)$ & $\mathrm{W}(2)-\mathrm{N}(2)$ & $1.746(6)$ \\
$\mathrm{W}(1)-\mathrm{C}(1)$ & $2.337(7)$ & $\mathrm{W}(2)-\mathrm{C}(24)$ & $2.338(8)$ \\
$\mathrm{W}(1)-\mathrm{C}(2)$ & $2.355(8)$ & $\mathrm{W}(2)-\mathrm{C}(25)$ & $2.353(8)$ \\
$\mathrm{W}(1)-\mathrm{C}(3)$ & $2.538(8)$ & $\mathrm{W}(2)-\mathrm{C}(26)$ & $2.525(8)$ \\
$\mathrm{W}(1)-\mathrm{C}(4)$ & $2.551(8)$ & $\mathrm{W}(2)-\mathrm{C}(27)$ & $2.531(8)$ \\
$\mathrm{W}(1)-\mathrm{C}(5)$ & $2.375(7)$ & $\mathrm{W}(2)-\mathrm{C}(28)$ & $2.343(8)$ \\
$\mathrm{Rh}(1)-\mathrm{S}(1)$ & $2.390(2)$ & $\mathrm{Rh}(2)-\mathrm{S}(3)$ & $2.396(2)$ \\
$\mathrm{Rh}(1)-\mathrm{S}(2)$ & $2.394(2)$ & $\mathrm{Rh}(2)-\mathrm{S}(4)$ & $2.385(2)$ \\
$\mathrm{N}(1)-\mathrm{O}(1)$ & $1.244(9)$ & $\mathrm{N}(2)-\mathrm{O}(2)$ & $1.255(9)$ \\
$\mathrm{W}(1)-\mathrm{S}(1)-\mathrm{Rh}(1)$ & $76.25(6)$ & $\mathrm{W}(2)-\mathrm{S}(3)-\mathrm{Rh}(2)$ & $75.44(6)$ \\
$\mathrm{W}(1)-\mathrm{S}(2)-\mathrm{Rh}(1)$ & $76.24(6)$ & $\mathrm{W}(2)-\mathrm{S}(4)-\mathrm{Rh}(2)$ & $75.86(6)$ \\
$\mathrm{W}(1)-\mathrm{N}(1)-\mathrm{O}(1)$ & $169.8(5)$ & $\mathrm{W}(2)-\mathrm{N}(2)-\mathrm{O}(2)$ & $170.1(5)$ \\
\hline
\end{tabular}




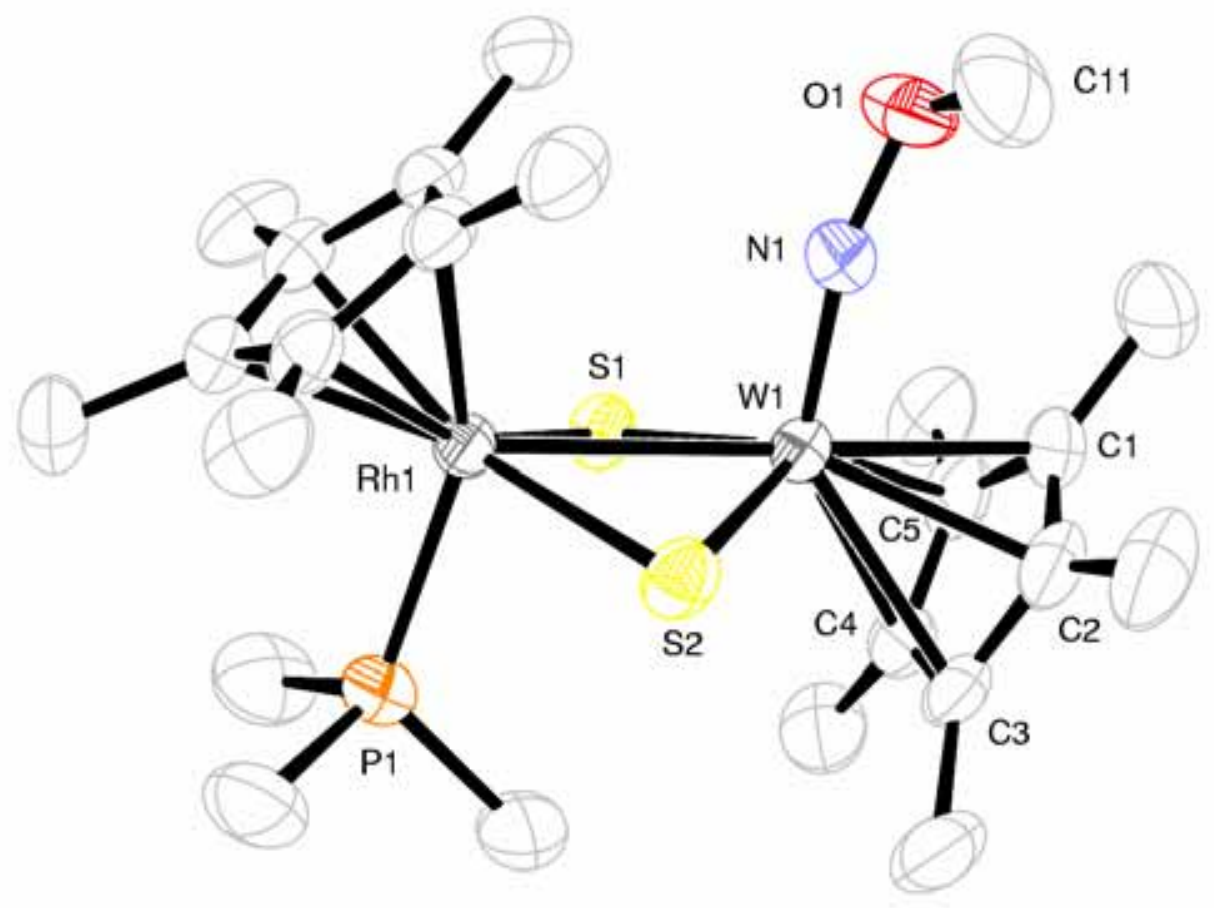

Figure S2. Molecular structure of the cationic part of $\left[\mathrm{Cp} * \mathrm{Rh}\left(\mathrm{PMe}_{3}\right)(\mu-\mathrm{S})_{2} \mathrm{~W}(\mathrm{NOMe}) \mathrm{Cp} *\right]\left(\mathrm{PF}_{6}\right)$ $\left(9^{+} \mathrm{PF}_{6}{ }^{-}\right)$. Thermal ellipsoids are shown at the $50 \%$ probability level. Hydrogen atom are omitted for clarity.

Table S4. Selected bond lengths $(\AA)$ and angles (deg) for $\mathbf{9}^{+} \mathrm{PF}_{6}{ }^{-}$.

\begin{tabular}{llll}
\hline $\mathrm{W}(1)-\mathrm{Rh}(1)$ & $2.9096(3)$ & $\mathrm{Rh}(1)-\mathrm{S}(1)$ & $2.388(1)$ \\
$\mathrm{W}(1)-\mathrm{S}(1)$ & $2.297(1)$ & $\mathrm{Rh}(1)-\mathrm{S}(2)$ & $2.398(1)$ \\
$\mathrm{W}(1)-\mathrm{S}(2)$ & $2.303(1)$ & $\mathrm{N}(1)-\mathrm{O}(1)$ & $1.350(6)$ \\
$\mathrm{W}(1)-\mathrm{N}(1)$ & $1.747(4)$ & $\mathrm{O}(1)-\mathrm{C}(11)$ & $1.436(7)$ \\
$\mathrm{W}(1)-\mathrm{C}(1)$ & $2.347(5)$ & $\mathrm{W}(1)-\mathrm{S}(1)-\mathrm{Rh}(1)$ & $76.74(4)$ \\
$\mathrm{W}(1)-\mathrm{C}(2)$ & $2.346(6)$ & $\mathrm{W}(1)-\mathrm{S}(2)-\mathrm{Rh}(1)$ & $76.46(4)$ \\
$\mathrm{W}(1)-\mathrm{C}(3)$ & $2.481(5)$ & $\mathrm{W}(1)-\mathrm{N}(1)-\mathrm{O}(1)$ & $164.0(3)$ \\
$\mathrm{W}(1)-\mathrm{C}(4)$ & $2.491(5)$ & $\mathrm{N}(1)-\mathrm{O}(1)-\mathrm{C}(11)$ & $112.3(4)$ \\
$\mathrm{W}(1)-\mathrm{C}(5)$ & $2.373(5)$ & & \\
\hline
\end{tabular}




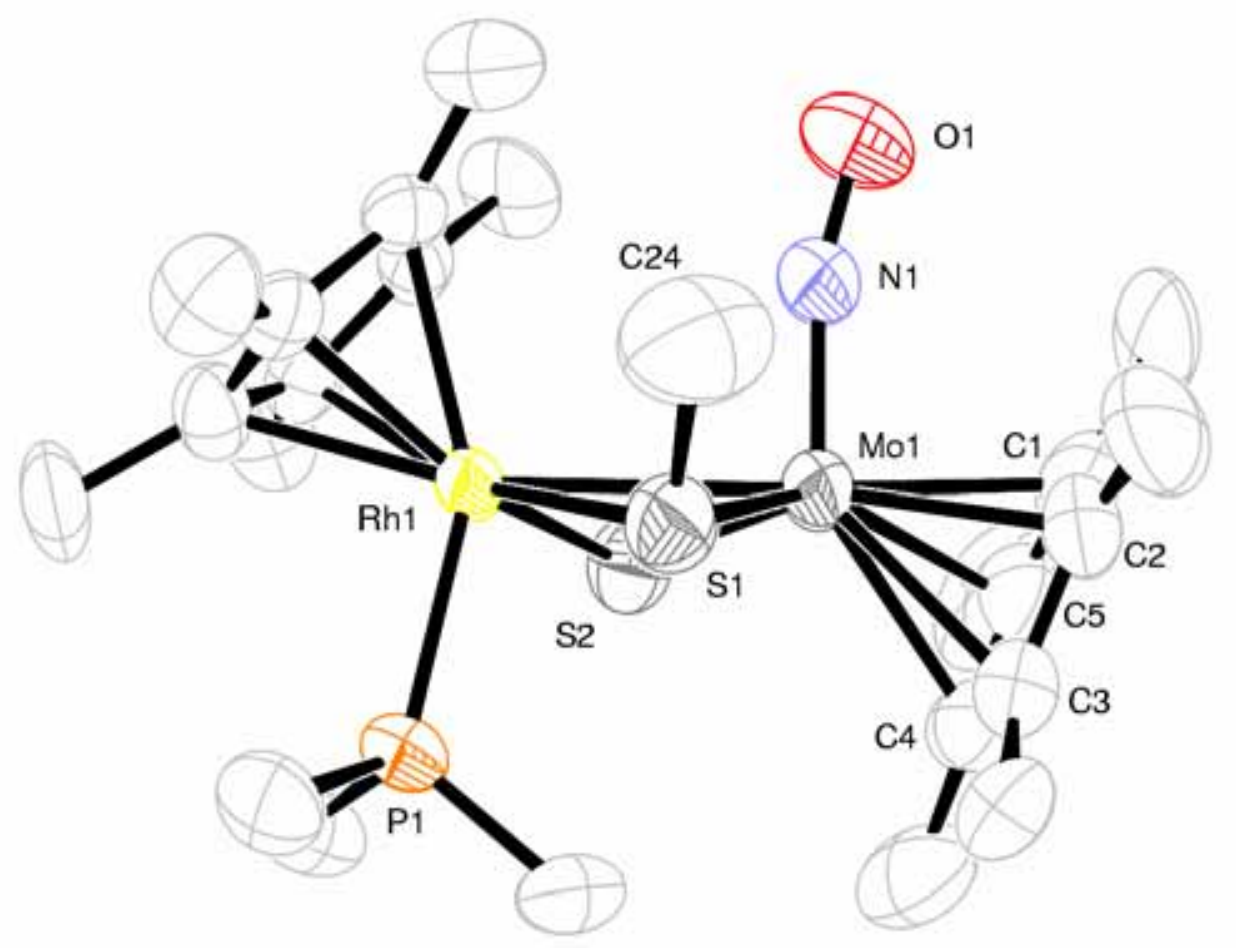

Figure S3. Molecular structure of the cationic part of [Cp*Rh(PMe 3$)(\mu-\mathrm{SMe})(\mu-\mathrm{S}) \mathrm{Mo}(\mathrm{NO}) \mathrm{Cp} *]-$ $\left(\mathrm{BPh}_{4}\right)\left(\mathbf{1 0}^{+} \mathrm{BPh}_{4}^{-}\right)$. One of the two crystallographically independent molecules. Thermal ellipsoids are shown at the 50\% probability level. Hydrogen atom are omitted for clarity.

Table S5. Selected bond lengths $(\AA)$ and angles (deg) for $\mathbf{1 0}^{+} \mathrm{BPh}_{4}$.

\begin{tabular}{llll}
\hline \multicolumn{3}{c}{ molecule 1} & \multicolumn{2}{c}{ molecule 2} \\
\hline $\mathrm{Mo}(1)-\mathrm{Rh}(1)$ & $2.940(1)$ & $\mathrm{Mo}(2)-\mathrm{Rh}(2)$ & $2.940(1)$ \\
$\mathrm{Mo}(1)-\mathrm{S}(1)$ & $2.385(3)$ & $\mathrm{Mo}(2)-\mathrm{S}(3)$ & $2.391(2)$ \\
$\mathrm{Mo}(1)-\mathrm{S}(2)$ & $2.317(3)$ & $\mathrm{Mo}(2)-\mathrm{S}(4)$ & $2.324(3)$ \\
$\mathrm{Mo}(1)-\mathrm{N}(1)$ & $1.76(1)$ & $\mathrm{Mo}(2)-\mathrm{N}(2)$ & $1.758(8)$ \\
$\mathrm{Mo}(1)-\mathrm{C}(1)$ & $2.33(1)$ & $\mathrm{Mo}(2)-\mathrm{C}(25)$ & $2.32(1)$ \\
$\mathrm{Mo}(1)-\mathrm{C}(2)$ & $2.31(1)$ & $\mathrm{Mo}(2)-\mathrm{C}(26)$ & $2.31(1)$ \\
$\mathrm{Mo}(1)-\mathrm{C}(3)$ & $2.43(1)$ & $\mathrm{Mo}(2)-\mathrm{C}(27)$ & $2.45(2)$ \\
$\mathrm{Mo}(1)-\mathrm{C}(4)$ & $2.47(2)$ & $\mathrm{Mo}(2)-\mathrm{C}(28)$ & $2.47(1)$ \\
$\mathrm{Mo}(1)-\mathrm{C}(5)$ & $2.39(1)$ & $\mathrm{Mo}(2)-\mathrm{C}(29)$ & $2.37(1)$ \\
$\mathrm{Rh}(1)-\mathrm{S}(1)$ & $2.395(3)$ & $\mathrm{Rh}(2)-\mathrm{S}(3)$ & $2.389(2)$ \\
$\mathrm{Rh}(1)-\mathrm{S}(2)$ & $2.400(3)$ & $\mathrm{Rh}(2)-\mathrm{S}(4)$ & $2.413(2)$ \\
$\mathrm{N}(1)-\mathrm{O}(1)$ & $1.22(1)$ & $\mathrm{N}(2)-\mathrm{O}(2)$ & $1.21(1)$ \\
$\mathrm{Mo}(1)-\mathrm{S}(1)-\mathrm{Rh}(1)$ & $75.92(9)$ & $\mathrm{Mo}(2)-\mathrm{S}(3)-\mathrm{Rh}(2)$ & $75.92(6)$ \\
$\mathrm{Mo}(1)-\mathrm{S}(2)-\mathrm{Rh}(1)$ & $77.10(9)$ & $\mathrm{Mo}(2)-\mathrm{S}(4)-\mathrm{Rh}(2)$ & $76.71(7)$ \\
$\mathrm{Mo}(1)-\mathrm{N}(1)-\mathrm{O}(1)$ & $164.9(9)$ & $\mathrm{Mo}(2)-\mathrm{N}(2)-\mathrm{O}(2)$ & $167.4(9)$ \\
\hline
\end{tabular}


